\title{
Bandwidth density optimization of misaligned optical interconnects
}

\author{
Hasan Aldiabat ${ }^{1}$, Nedal Al-ababneh ${ }^{2}$ \\ ${ }^{1}$ Department of Telecommunications Engineering, Yarmouk University, Irbid, Jordan \\ ${ }^{2}$ Department of Electrical Engineering, Jordan University of Science and Technology, Ar-Ramtha, Jordan
}

\begin{tabular}{l} 
Article Info \\
\hline Article history: \\
Received Apr 30, 2021 \\
Revised Aug 30, 2021 \\
Accepted Sep 28, 2021 \\
\hline
\end{tabular}

Keywords:

Bandwidth density

Error correction codes

Optical crosstalk noise

Optical interconnects

Wireless communications

\begin{abstract}
In this paper, the bandwidth density of misaligned free space optical interconnects (FSOIs) system with and without coding under a fixed bit error rate is considered. In particular, we study the effect of using error correction codes of various codeword lengths on the bandwidth density and misalignment tolerance of the FSOIs system in the presence of higher order modes. Moreover, the paper demonstrates the use of the fill factor of the detector array as a design parameter to optimize the bandwidth density of the communication. The numerical results demonstrate that the bandwidth density improves significantly with coding and the improvement is highly dependent on the used codeword length and code rate. In addition, the results clearly show the optimum fill factor values that achieve the maximum bandwidth density and misalignment tolerance of the system.
\end{abstract}

This is an open access article under the CC BY-SA license.

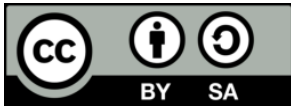

\section{Corresponding Author:}

Hasan Aldiabat

Department of Telecommunications Engineering, Yarmouk University

Shafiq Irshidat st. 21163, Irbid, Jordan

Email: hasan.aldiabat@yu.edu.jo

\section{INTRODUCTION}

Optical interconnects were proposed to relieve the predicted bottleneck in many high-speed digital computing systems in the era of big data. This is due to their high capacity, high speed, wide bandwidth, and low power consumption [1]-[3]. Different alternatives of optical interconnect technologies can be evaluated using the capacity which is directly related to the bandwidth density. The interconnect capacity is defined as the time space density product evaluated at a fixed operating power [4]-[7]. A significantly large interconnect capacity can be achieved by using a number of spatial channels of free space optical interconnects (FSOIs) [8], [9]. FSOIs using vertical cavity service emitting lasers (VCSEL) have witnessed exponential growth in vital applications, including data communications, optical sensing, laser printing, and illumination [10]-[14].

Like any wireless communication channel, FSOI channels suffer from noise [15]-[17]. In this paper, we consider two main sources of noise which are the crosstalk among the channels and the detector amplifier thermal noise. Crosstalk arises from the laser beam divergence, and it increases with the density of the channels. On the other hand, thermal noise, which is characterized by a noise equivalent power (NEP), arises due to the large bandwidth of the FSOI channels [4], [18]-[21]. These two types of noise directly affect the speed and bandwidth density of optical channels; hence, the use of error correction coding schemes is proposed to combat the noise effects. Several researchers have investigated the use of error correction codes (ECC) to optimize the time and space resources of FSOI systems. Neifeld and Kostuk [18] analyzed the use of Reed Solomon codes to optimize the channel spacing and data rates of optical interconnects. The results were demonstrated for both rectangular aperture and Gaussian propagation models. However, the authors overestimated the crosstalk noise power in their work which led to suboptimum findings. Al-Ababneh and 
Tawalbeh [22] studied the effect of fill factor of the photodetector array and the code rate of the ECCs on the bandwidth density of FSOI channels. It presented analytical and simulation results that demonstrate the importance of optimizing the fill factor which is defined as the ratio of the used detector diameter over the interconnect spacing. This ratio significantly affects the noise and signal powers, so the bandwidth density. The implementation of extended Golay codes to model forward error correction in the operation of optical data links is investigated in [23]. The authors used Golay codes to compensate for a single and multiple inoperative sublinks as erasures. Jun et al. [21] made a comparison among a variety of error control schemes, including error detection (ED) and error correction (EC) schemes, that can be used in optical interconnects. The comparison was made in terms of area, latency, and power consumption. In this paper, we study the performance of FSOIs system model in the presence of, besides the fundamental mode, higher order transverse modes. In particular, the effect of interconnect spacing, signal to noise ratio (SNR), and fill factor (FF) on the bandwidth density of the FSOIs system is presented. In addition, the impact of using ReedSolomon ECCs (RS-ECCs) of several codeword lengths on the bandwidth density and misalignment between the transmitter and receiver arrays is considered. We use the RS-ECCs at the optimum code rate of every used codeword length. The rest of the paper is organized as follows: in section 2, we provide a description of the FSOIs system and derivations of the bandwidth density of the fundamental and the higher order transverse modes. The performance of the studied transverse modes with respect to channel spacing, SNR, misalignment, and coding is provided in section 3. Finally, the conclusions and future works are drawn in section 4.

\section{RESEARCH METHODS}

For the purpose of the study presented in this paper, we consider an array of nine elements of vertical cavity surface emitting lasers (VCSELs) as a transmitter and an array of nine photodetectors as a receiver. The interconnect distance between the arrays is set to be D in Figure 1(a). The VCSELs are arranged as shown in Figure 1(b), where A is the VCSELs' diameter and B, shown in Figure 1(c), is the photodetectors' diameter. The $\Delta$ parameter represents the distance between any two consecutive photodetectors (PDs) and is called channel spacing, where the ratio between $\mathrm{B}$ and $\Delta$ is defined as the fill factor (FF) parameter.

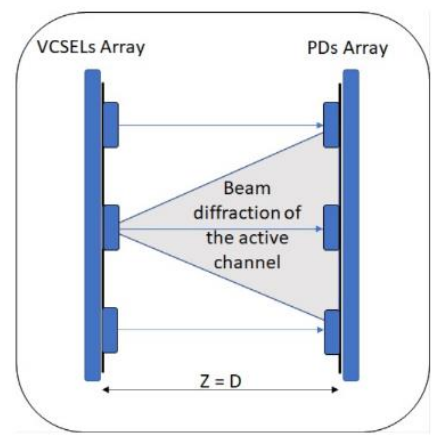

(a)

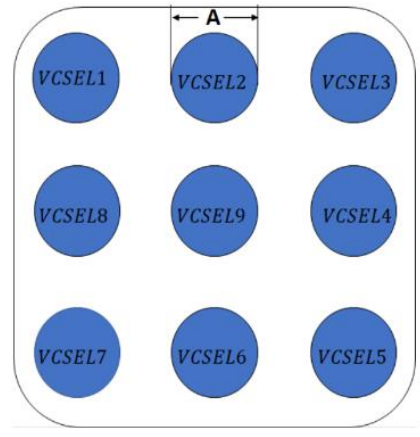

(b)

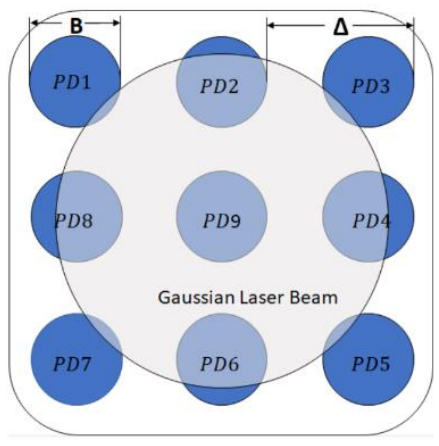

(c)

Figure 1. Two-dimensional schematic representation of (a) the FSOIs system model, (b) VCSELs array, and (c) photodetectors array 
In this paper, we have assumed that the parameters of all VCSELs are the same. Moreover, we consider only the two major noise sources that are the optical crosstalk noise and detector amplifier thermal noise. The optical crosstalk noise results from the divergence nature of the laser beam as illustrated in Figures 1(a) and 1(c), while the detector amplifier thermal noise which is also known as the noise equivalent power (NEP) characterizes the employed photodetectors. As stated in [22], the bandwidth density (BD) of FSOI channels depends on the ratio of the data rate $(\mathrm{R})$ of a single optical interconnect and the channel spacing $(\Delta)$ as $(1)$ :

$$
\mathrm{BD}=\frac{\mathrm{R}}{\Delta^{2}},
$$

where $\mathrm{R}$ is chosen such that the transmitter (modulator) can achieve this rate and the receiver (detector) can demodulate the laser beam efficiently. The relationship between the BD and signal to noise ratio (SNR) as well as other parameters of FSOIs system can be explored from the definition of R, which is defined as (20) [22],

$$
\mathrm{R}=\frac{\left(P_{o} \times S-P_{o} \times N \times \mathrm{SNR}\right)^{2}}{S N R^{2} \times \mathrm{NEP}^{2}}
$$

where $P_{o}$ is the transmitted power per optical channel in watt, $S$ is the useful received optical signal by intended receiver, $N$ is the optical crosstalk noise, and NEP is the noise equivalent power in watt per square root of Hertz. The definition of $\mathrm{R}$ in the above equation is derived based on the definition of SNR given by (3) [22].

$$
\mathrm{SNR}=10 \log \left(\frac{P_{o} \times S}{P_{o} \times N+\mathrm{NEP} \times R^{1 / 2}}\right) .
$$

It is clear from (1) and (2) that the BD can be found by finding the values of the optical signal power (S) and the optical crosstalk noise $(N)$ for predetermined values of $P_{o}$, NEP, SNR, and $\Delta$. The value of $\mathrm{S}$ received by the photodetector PD9 in Figure 1(c) from the VCSEL9 in Figure 1(b) is determined by integrating the two-dimensional normalized optical field over the surface of PD9 which is located at plane $\mathrm{Z}=D$ from the VCSELs array. If we assume that the VCSEL9 is operating in the transverse electromagnetic mode $m n\left(\mathrm{TEM}_{m n}\right)$, where $m$ and $n$ are integers that represent the radial and angular mode orders, respectively, then $\mathrm{S}$ can be determined as (4).

$$
S_{m n}=\iint_{P D_{9}} I_{m n}(r, \theta, D) r \mathrm{~d} r \mathrm{~d} \theta=\int_{0}^{2 \pi} \int_{0}^{\frac{\Delta}{2}} I_{m n}(r, \theta, D) r \mathrm{~d} r \mathrm{~d} \theta
$$

With the help of [19], the normalized optical field (I) of a transverse mode $\mathrm{TEM}_{\mathrm{mn}}$ at a distance $D$ from the transmitter is defined as (5),

$$
I_{m n}(r, \theta, D)=\frac{4 m !}{\pi \times W_{D}^{2} \times(m+n) ! \times\left(\delta_{0 n}+1\right)} \times\left(\frac{\sqrt{2} r}{W_{D}}\right)^{2 n} \times\left(L_{m}^{n}\left(\frac{2 r^{2}}{W_{D}^{2}}\right)\right)^{2} \times \exp \left(\frac{-2 r^{2}}{W_{D}^{2}}\right) \times \cos ^{2}(n \theta),
$$

where $W_{D}$ is the laser beam spot size at the photodetector that is away distance $D$ from the VCSELs array, and is given by:

$$
W_{D}=W_{i} \sqrt{\left(\frac{2 \pi \times \lambda \times D}{A^{2}}\right)^{2}+1}
$$

where $W_{i}$ and $\lambda$ are the beam waist at the output of the VCSEL which is equal to $A /(\sqrt{2} \pi)$ and the operating wavelength, respectively. The $\delta_{0 n}$ function is defined as:

$$
\delta_{0 n}=\left\{\begin{array}{lc}
1, & n=0 \\
0, & \text { otherwise }
\end{array},\right.
$$

and $L_{m}^{n}(\alpha)$ is a Laguerre polynomial of $\operatorname{order}(m, n)$ and is given by [24],

$$
L_{m}^{n}(\alpha)=\frac{\alpha^{-n}}{m !} \times \exp (\alpha) \times \frac{\mathrm{d}^{m}}{\mathrm{~d} \alpha^{m}}\left(\alpha^{m+n} \times \exp (-\alpha)\right) .
$$


In this paper, we consider three modes of operation for the laser beam which are the TEM ${ }_{00}, \mathrm{TEM}_{10}$, and $\mathrm{TEM}_{20}$. Moreover, we assume that there is no coupling among the laser modes. The normalized optical field (5) of the aforementioned transverse modes, respectively, is given as (6) to (8),

$$
\begin{aligned}
& I_{00}=\frac{2}{\pi \times W_{D}^{2}} \times \exp \left(\frac{-2 r^{2}}{W_{D}^{2}}\right) \times\left(L_{0}^{0}\left(\frac{2 r^{2}}{W_{D}^{2}}\right)\right)^{2}, \\
& I_{10}=\frac{2}{\pi \times W_{D}^{2}} \times \exp \left(\frac{-2 r^{2}}{W_{D}^{2}}\right) \times\left(L_{1}^{0}\left(\frac{2 r^{2}}{W_{D}^{2}}\right)\right)^{2},
\end{aligned}
$$

and

$$
I_{20}=\frac{2}{\pi \times W_{D}^{2}} \times \exp \left(\frac{-2 r^{2}}{W_{D}^{2}}\right) \times\left(L_{2}^{0}\left(\frac{2 r^{2}}{W_{D}^{2}}\right)\right)^{2},
$$

where $L_{0}^{0}(\alpha)=1, L_{1}^{0}(\alpha)=1-\alpha$, and $L_{2}^{0}(\alpha)=1-2 \alpha+(1 / 2) \alpha^{2}$.

Upon substituting the $I_{m, n}$ of (6), (7), and (8) into (4), we find the optical signal power $(S)$ received by the intended photodetector, PD9, due to the operation of each mode separately. The optical crosstalk noise $(N)$ which results from the divergence of the laser beam, as shown in Figure 1(a) and Figure 1(c), can be calculated based on the power falling from the VCSEL9 on the photodetectors PD1, PD2, PD3, PD4, PD5, PD6, PD7, and PD8. For the fundamental order Gaussian mode, TEM 00 , the $N_{00}$ can be defined as (9),

$$
\begin{aligned}
& N_{00}=\frac{2}{\pi \times W_{D}^{2}} \sum_{j=1}^{8} \iint_{\mathrm{PD}_{j}} \exp \left(\frac{-2 r^{2}}{W_{D}^{2}}\right) r \mathrm{~d} r \mathrm{~d} \theta= \\
& \frac{4 \times 2}{\pi \times W_{D}^{2}} \int_{0}^{2 \pi} \int_{0}^{\frac{\Delta}{2}} \exp \left(\frac{-2 r_{1}^{2}}{W_{D}^{2}}\right) r \mathrm{~d} r \mathrm{~d} \theta+\frac{4 \times 2}{\pi \times W_{D}^{2}} \int_{0}^{2 \pi} \int_{0}^{\frac{\Delta}{2}} \exp \left(\frac{-2 r_{2}^{2}}{W_{D}^{2}}\right) r \mathrm{~d} r \mathrm{~d} \theta,
\end{aligned}
$$

where $r_{1}^{2}=(r \cos (\theta)-\Delta)^{2}+(r \sin (\theta))^{2}$ and $r_{2}^{2}=(r \cos (\theta)-\Delta)^{2}+(r \sin (\theta)+\Delta)^{2}$. In (9), we exploited the fact that the optical crosstalk noise received by the photodetectors PD1, PD3, PD5, and PD7 is the same, as well as the one received by the photodetectors PD2, PD4, PD6, and PD8 is the same. This is due to the symmetry in the distribution of the photodetectors and the rotational symmetry of the laser beam.

For the $\mathrm{TEM}_{10}$ and $\mathrm{TEM}_{20}$ modes, the $N$ of each of these modes can be determine as (10) and (11):

$$
\begin{aligned}
& N_{10}=\frac{2}{\pi \times W_{D}^{2}} \sum_{j=1}^{8} \iint_{\mathrm{PD}_{j}} \exp \left(\frac{-2 r^{2}}{W_{D}^{2}}\right) \times\left(1-\frac{2 r^{2}}{W_{D}^{2}}\right)^{2} r \mathrm{~d} r \mathrm{~d} \theta= \\
& +\frac{4 \times 2}{\pi \times W_{D}^{2}} \int_{0}^{2 \pi} \int_{0}^{\frac{\Delta}{2}} \exp \left(\frac{-2 r_{2}^{2}}{W_{D}^{2}}\right) \times\left(1-\frac{2 r_{2}^{2}}{W_{D}^{2}}\right)^{2} r \mathrm{~d} r \mathrm{~d} \theta, \\
& \frac{4 \times 2}{\pi \times W_{D}^{2}} \int_{0}^{2 \pi} \int_{0}^{\frac{\Delta}{2}} \exp \left(\frac{-2 r_{1}^{2}}{W_{D}^{2}}\right) \times\left(1-\frac{2 r_{1}^{2}}{W_{D}^{2}}\right)^{2} r \mathrm{~d} r \mathrm{~d} \theta
\end{aligned}
$$

and

$$
\begin{aligned}
& N_{20}=\frac{2}{\pi \times W_{D}^{2}} \sum_{j=1}^{8} \iint_{\mathrm{PD}_{j}} \exp \left(\frac{-2 r^{2}}{W_{D}^{2}}\right) \times\left(1-\frac{4 r^{2}}{W_{D}^{2}}+0.5\left(\frac{2 r^{2}}{W_{D}^{2}}\right)^{2}\right)^{2} r \mathrm{~d} r \mathrm{~d} \theta= \\
& +\frac{4 \times 2}{\pi \times W_{D}^{2}} \int_{0}^{2 \pi} \int_{0}^{\frac{\Delta}{2}} \exp \left(\frac{-2 r_{2}^{2}}{W_{D}^{2}}\right) \times\left(1-\frac{4 r_{2}^{2}}{W_{D}^{2}}+0.5\left(\frac{2 r_{2}^{2}}{W_{D}^{2}}\right)^{2}\right)^{2} r \mathrm{~d} r \mathrm{~d} \theta, \\
& \frac{4 \times 2}{\pi \times W_{D}^{2}} \int_{0}^{2 \pi} \int_{0}^{\frac{\Delta}{2}} \exp \left(\frac{-2 r_{1}^{2}}{W_{D}^{2}}\right) \times\left(1-\frac{4 r_{1}^{2}}{W_{D}^{2}}+0.5\left(\frac{2 r_{1}^{2}}{W_{D}^{2}}\right)^{2}\right)^{2} r \mathrm{~d} r \mathrm{~d} \theta
\end{aligned}
$$

where $r_{1}^{2}$ and $r_{2}^{2}$ are same as the ones that are defined in (9).

Equations (4), (9), (10), and (11) can be solved numerically or by following analytical models to solve integrals including the ones proposed in [16]. Having determined $S_{m n}$ and $N_{m n}$ of each transverse mode, the BD can be obtained for any given SNR level and a predetermined values of $P_{o}$ and NEP. The BD 
of the FSOIs system and the misalignment tolerance between the transmitter (VCSELs) and the receiver (PDs) arrays can be significantly improved with coding. For this purpose, we introduce the use of error correction codes (ECCs) in the considered FSOIs system. In particular, we use Reed-Solomon (RS) ECCs of various codeword lengths to demonstrate the effect of coding on the FSOIs system [25]. The effect mainly reduces the SNR level required to achieve a predetermined bit error rate (BER) of the system. The BER is defined as in (12) [21], [22], [26], [27]:

$$
\mathrm{BER}=\frac{1}{2}\left(1-\operatorname{erf}\left(\frac{1}{2} \sqrt{\frac{\mathrm{SNR}}{2}}\right)\right)
$$

where erf in (12) is the error function defined as $\operatorname{erf}(x)=\frac{2}{\sqrt{\pi}} \int_{0}^{x} \exp \left(-y^{2}\right) \mathrm{d} y$.

The reduction in the SNR level due to coding is translated into a gain in the bandwidth density, and this gain is given by [18],

$$
G_{B D}=\frac{B D_{e f}-B D_{u}}{B D_{u}}
$$

where $\mathrm{BD}_{u}$ and $\mathrm{BD}_{e f}$ are the uncoded bandwidth density and the effective bandwidth density, respectively. The $\mathrm{BD}_{e f}$ is defined as the code rate ( $\mathrm{r}$ ) multiplied by the coded $\mathrm{BD}_{c}$, where $\mathrm{r}$ is the proportion of useful information bits of each generated codeword.

\section{RESULTS AND DISCUSSION}

In this section, we present how design parameters of FSOIs system affect the BD and the misalignment tolerance. In particular, we demonstrate the effect of channel spacing, SNR, fill factor (FF), and coding on the BD and misalignment tolerance on the system. The design parameters of the simulated FSOIs system presented in this paper are set to be as the following: operating wavelength $(\lambda)=0.85 \mu \mathrm{m}$, transmitted power per channel $\left(P_{o}\right)=50 \mu \mathrm{W}$, interconnect distance $(D)=20 \mathrm{~mm}$, VCSELs' diameter $(\mathrm{A})=0.03$ $\mathrm{mm}$, signal to noise ratio $(\mathrm{SNR})=10 \mathrm{~dB}$, and noise equivalent power $(\mathrm{NEP})=0.1 \mathrm{nW} / \sqrt{\mathrm{H}_{z}}$. All results shown in this section are based on these aforementioned parameters.

Figure 2 demonstrates the bandwidth density (BD) of the $\mathrm{TEM}_{00}, \mathrm{TEM}_{10}$, and $\mathrm{TEM}_{20}$ operating modes as a function of channel spacing using FF values of $0.8(\mathrm{~B}=0.8 \Delta$, where $\mathrm{B}$ and $\Delta$ are the photodetectors' diameter and channel spacing, respectively), and $1(\mathrm{~B}=\Delta)$. From the results shown in the figure, we can see that there is an optimal channel spacing $\left(\Delta_{o p t}\right)$ for each FF of each studied TEM mode. For TEM $_{o 0}$ mode as shown in Figure 2(a), the $\Delta_{o p t}$ that achieves the maximum BD at FF of 0.8 is $0.4 \mathrm{~mm}$ and at FF of 1 is $0.44 \mathrm{~mm}$. At small values of channel spacing $\left(<2.6 \times 10^{-4} \mathrm{~m}\right)$, the BD is very small compared to the optimal BD, therefore, it did not appear on the figure. For TEM $\mathrm{T}_{10}$, the values of $\Delta_{\text {opt }}$ at FF values of 0.8 and 1 are $0.67 \mathrm{~mm}$ and $0.65 \mathrm{~mm}$, respectively, as demonstrated in Figure 2(b). Figure 2(c) shows the results of $\mathrm{TEM}_{20}$ operating mode. It is clear from the Figure that $\Delta_{\text {opt }}$ at FF of 1 achieves higher BD than $\Delta_{o p t}$ at FF of 0.8. In addition, the figure shows that the values of $\Delta_{o p t}$ at FF values of 0.8 and 1 are $0.89 \mathrm{~mm}$ and 0.81 $\mathrm{mm}$, respectively. The dependence of $\mathrm{BD}$ on the FF is considered in Figure 3. It is clear from the figure that there is an optimum FF value that can achieve a maximum BD for each operating mode. For instance, the optimum FF value of $\mathrm{TEM}_{10}$ mode is 0.93 which allows the BD of the FSOIs system to be as high as $5.03 \times 10^{15} \mathrm{bit} / \mathrm{sec} . / \mathrm{m}^{2}$.

Figure 4 depicts the $\mathrm{BD}$ as a function of SNR levels. The BD of the considered operating modes is computed at the optimal value of channel spacing using an FF of 0.8 for each mode, see Figure 2. It is obvious from the findings that the BD is highly related to the used SNR level and can be approximated by the relation $R \propto 1 / S_{N R}{ }^{2}$. This suggests the use of ECCs to improve the performance of FSOIs systems in terms of BD. Figure 5(a) demonstrates the coding gain of RS-ECCs of various codeword lengths as a function of the code rate. The main idea of exploiting coding gain to improve the BD is revealed by the following example. If we consider a codeword of length 127 and a code rate of 0.64 , then from Figure 5(a) we find that the coding gain is 2.7. This means that the SNR used to generate the results of Figure 2 can be reduced from 10 to $3.7,10 \div 2.7=3.7$, while maintaining the same BER. As mentioned in (13), this reduction in the SNR level is turned into a BD gain. Figure 5(b) shows the correspondence between the BD gain and the code rate for five codeword lengths with $\mathrm{SNR}_{\text {uncoded }}=10 \mathrm{~dB}$. It is clear from the results that the coding rate is a crucial parameter which directly affects the BD gain due to coding. 


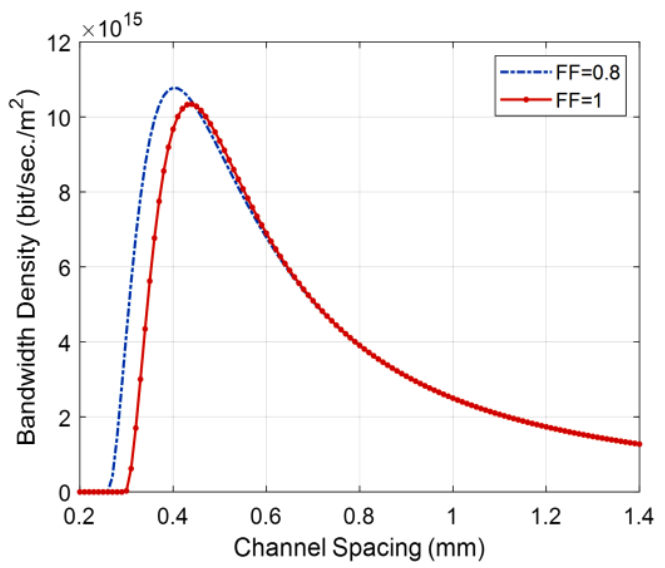

(a)

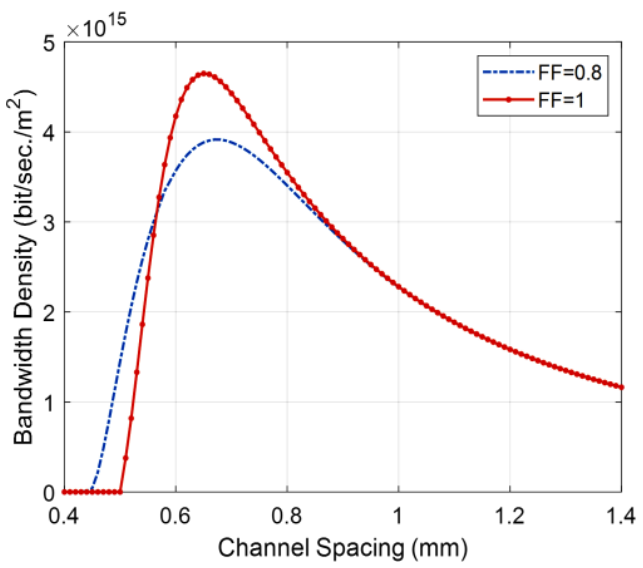

(b)

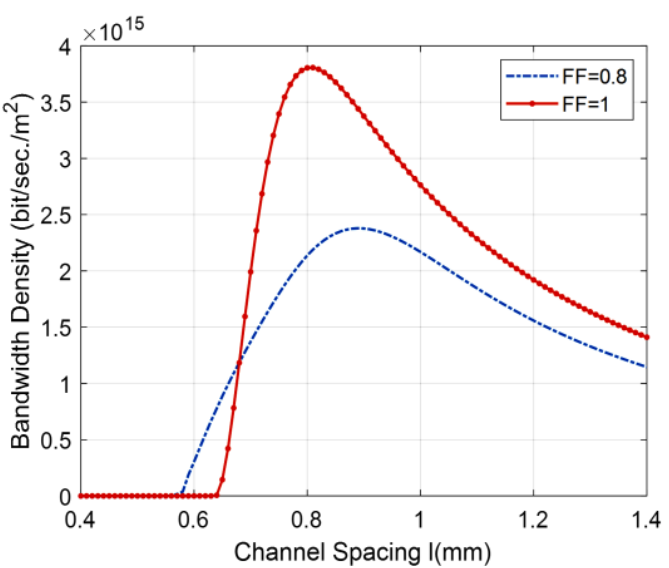

(c)

Figure 2. Bandwidth density versus channel spacing using FF values of 0.8 and 1 for (a) TEM 00 , (b) TEM 10 , and (c) $\mathrm{TEM}_{20}$ operation modes

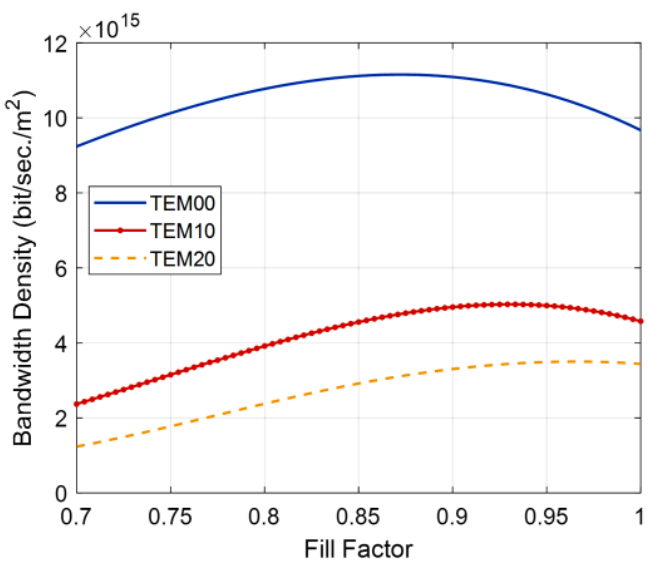

Figure 3. Bandwidth density of TEM00, TEM10, and TEM20 operating modes versus fill factor

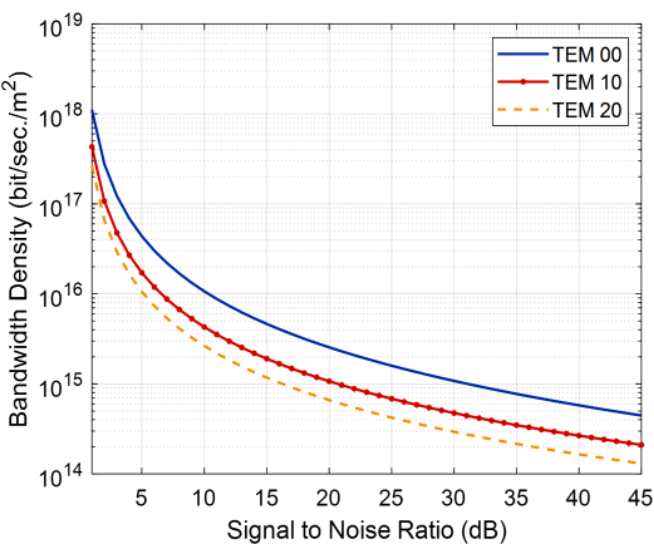

Figure 4. Bandwidth density as a function of signal to noise ratio (SNR)

The effect of misalignment between the VCSEL array and photodetector array on the BD with and without coding using an FF of 0.8 is considered in Figure 6. As expected, the use of RS-ECCs improves the $\mathrm{BD}$ of the FSOIs system of all operating modes. The improvements vary based on the used codeword length, where we note that as the codeword length increased, the improvement in the BD also gets increased. 
However, the misalignment tolerance at the used FF is the same before and after coding. For example, the $\mathrm{BD}$ of $\mathrm{TEM}_{00}$ mode Figure 6(a) when the misalignment is zero is identical to the BD when the misalignment is $1.14 \mathrm{~mm}$ before and after coding. The results of $\mathrm{TEM}_{10}$ and $\mathrm{TEM}_{20}$ exhibit the same performance as $\mathrm{TEM}_{00}$ and are shown in Figures 6(b) and 6(c), respectively. These results are new and have not been reported in the literature before.

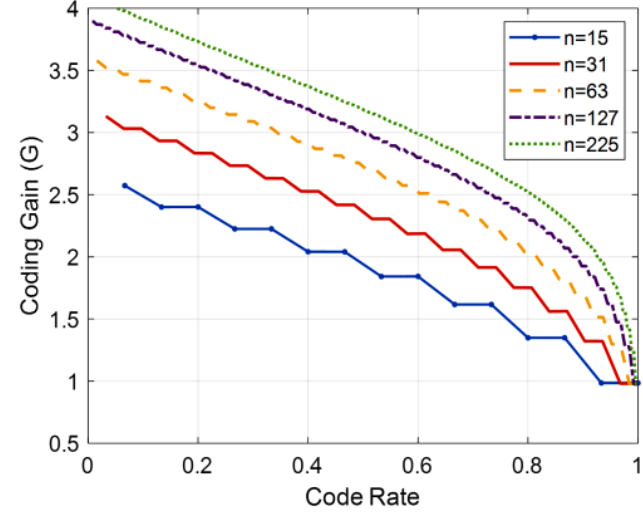

(a)

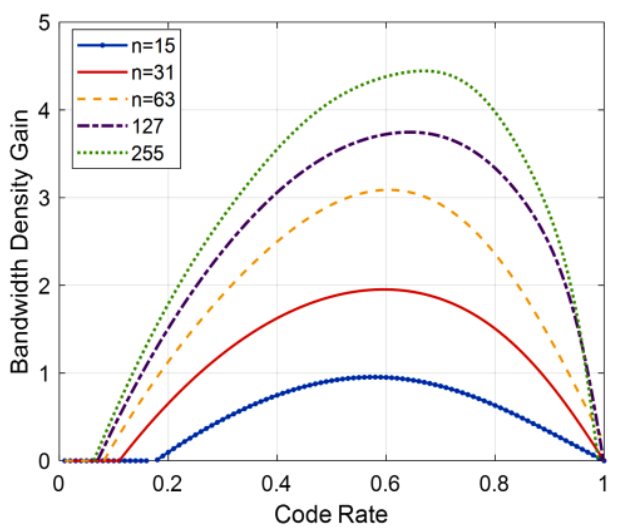

(b)

Figure 5. RS-ECC of codeword lengths 15, 31, 63, 127, and 255 effects on (a) coding gain and (b) bandwidth density for a BER of $10^{-12}$

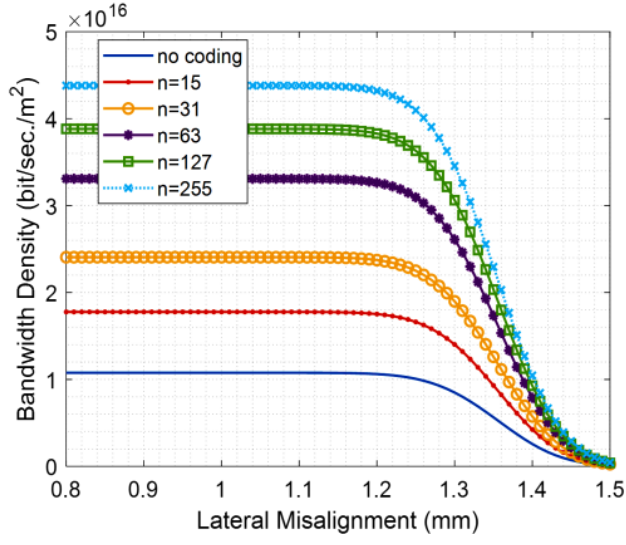

(a)

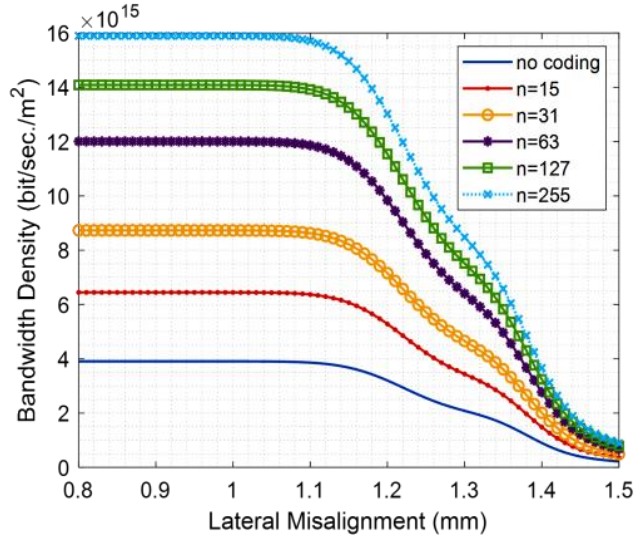

(b)

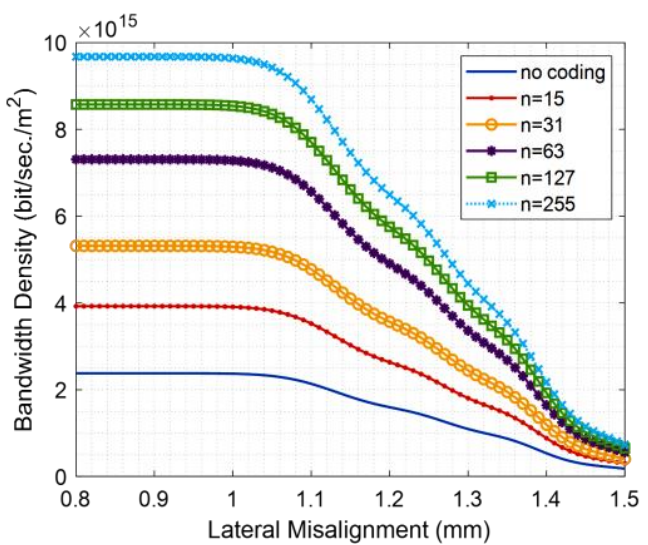

(c)

Figure 6. Bandwidth density versus lateral misalignment before and after applying RS-ECC of codeword lengths $15,31,63,127$, and 255 for (a) $\mathrm{TEM}_{00}$, (b) $\mathrm{TEM}_{10}$, and (c) $\mathrm{TEM}_{20}$ operating modes 
In order to examine the effect of the FF on the misalignment with and without coding, we generated the results shown in Figure 7. One can see from the results that as the FF increases the misalignment tolerance to achieve a given interconnect capacity also increases, but this continues until reaching an optimum FF. Beyond the optimum FF range, the misalignment tolerance starts decreasing. This behavior is common for all examined modes of operation, TEM $_{00}$ in Figure 7(a), TEM 10 in Figure 7(b), and TEM $_{20}$ in Figure 7(c). Exploiting RS-ECCs improves the misalignment tolerance of the FSOIs system as demonstrated in Figure 7. It is clear from the figure as the codeword length increased, the misalignment tolerance of the system over the optimum FF range also increased for some modes. Table 1 summarizes the results of the optimum FF ranges and the misalignment tolerances of the studied modes before and after coding of various codeword lengths. It is worth mentioning that these results are new and have not been published in the literature before.

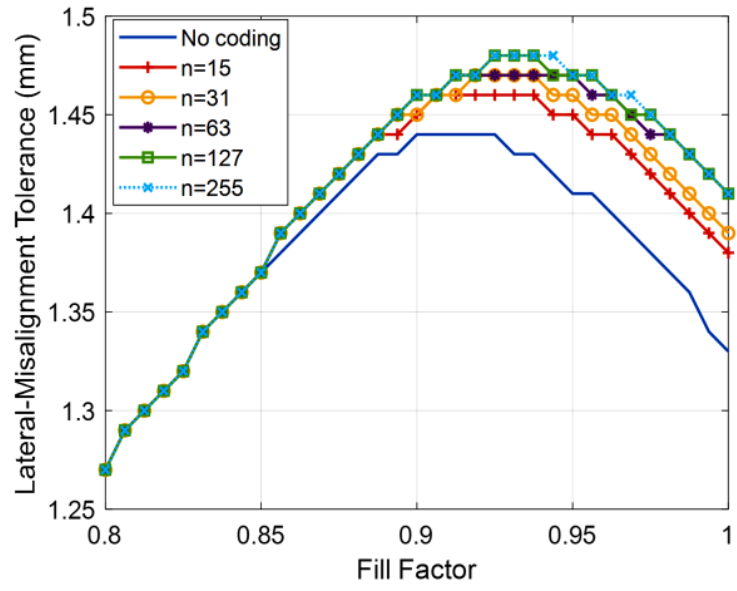

(a)

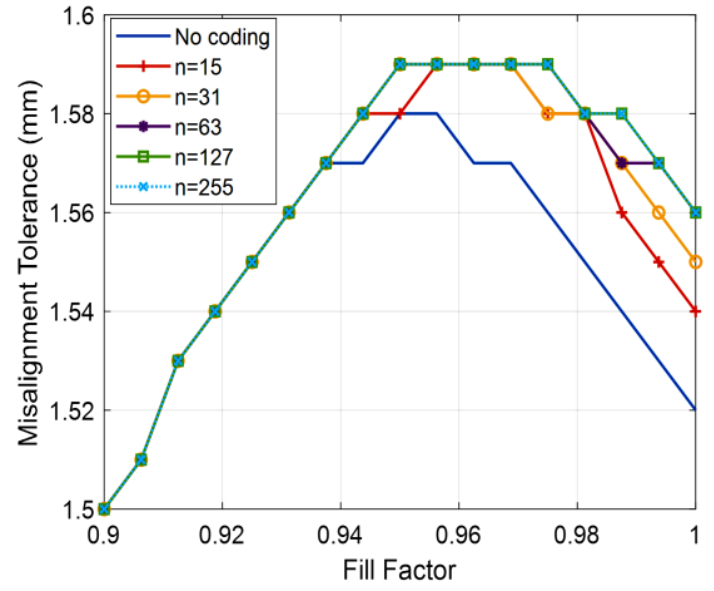

(b)

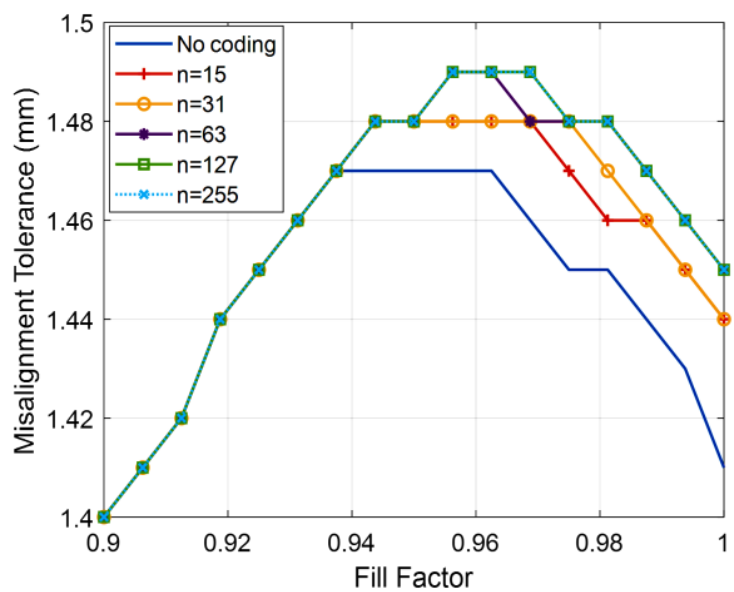

(c)

Figure 7. Misalignment tolerance as a function of FF before and after applying RS-ECC of codeword lengths $15,31,63,127$, and 255 for (a) $\mathrm{TEM}_{00}$, (b) $\mathrm{TEM}_{10}$, and (c) $\mathrm{TEM}_{20}$ operating modes

Table 1. Optimum FF range of FSOIs system for maximum misalignment tolerance in mm

\begin{tabular}{ccccccc}
\multicolumn{7}{c}{ before and after coding } \\
\hline $\begin{array}{c}\text { Codeword length } \\
(\mathrm{n})\end{array}$ & Opt. FF & Max. misal. & Opt. FF & Max. misal. & Opt. FF & Max. misal \\
\hline- & $0.900-0.925$ & 1.44 & $0.950-0.956$ & 1.58 & $0.937-0.963$ & 1.47 \\
15 & $0.906-0.938$ & 1.46 & $0.956-0.969$ & 1.59 & $0.944-0.969$ & 1.48 \\
31 & $0.919-0.938$ & 1.47 & $0.950-0.969$ & 1.59 & $0.944-0.975$ & 1.48 \\
63 & $0.919-0.950$ & 1.47 & $0.950-0.975$ & 1.59 & $0.956-0.963$ & 1.49 \\
127 & $0.925-0.938$ & 1.48 & $0.950-0.975$ & 1.59 & $0.956-0.969$ & 1.49 \\
255 & $0.925-0.944$ & 1.48 & $0.950-0.975$ & 1.59 & $0.956-0.969$ & 1.49 \\
\hline
\end{tabular}




\section{CONCLUSION}

In this paper, an analytical model for the bandwidth density of a short range FSOIs system with and without coding at a given BER is provided. In particular, we demonstrate the effect coding and fill factor on the performance of bandwidth density and misalignment tolerance of the FSOIs system. Both the fundamental order Gaussian mode of the laser beam and higher order transverse modes have been considered in the system design. The results demonstrate that bandwidth density and misalignment tolerance could be optimized by selecting appropriate code rate and fill factor levels. This study can be extended to include the rectangular propagation model of the laser beam and to consider lenses in the design of the FSOIs system. In addition, different types of coding schemes can be considered.

\section{REFERENCES}

[1] K. H. Koo, H. Cho, P. Kapur, and K. C. Saraswat, "Performance comparisons between carbon nanotubes, optical, and Cu for future high-performance on-chip interconnect applications," IEEE Transactions on Electron Devices, vol. 54, no. 12, pp. 3206-3215, 2007, doi: 10.1109/TED.2007.909045.

[2] A. Liu, P. Wolf, J. A. Lott, and D. Bimberg, "Vertical-cavity surface-emitting lasers for data communication and sensing," Photonics Research, vol. 7, no. 2, pp. 121-136, Jan. 2019, doi: 10.1364/prj.7.000121.

[3] J. A. Kash et al., "Optical interconnects in exascale supercomputers," in 2010 23rd Annual Meeting of the IEEE Photonics Society, PHOTINICS 2010, Nov. 2010, pp. 483-484, doi: 10.1109/PHOTONICS.2010.5698971.

[4] W. Hu, X. Li, J. Yang, and D. Kong, "Crosstalk analysis of aligned and misaligned free-space optical interconnect systems," Journal of the Optical Society of America A, vol. 27, no. 2, pp. 200-205, Jan. 2010, doi: 10.1364/josaa.27.000200.

[5] C. Fan et al., "Digital free-space optical interconnections: a comparison of transmitter technologies," Applied Optics, vol. 34, no. 17, pp. 3103-3115, Jun. 1995, doi: 10.1364/ao.34.003103.

[6] M. R. Feldman, C. C. Guest, T. J. Drabik, and S. C. Esener, "Comparison between electrical and free space optical interconnects for fine grain processor arrays based on interconnect density capabilities," Applied Optics, vol. 28, no. 18, pp. 3820-3829, Sep. 1989, doi: 10.1364/AO.28.003820.

[7] N. Al-Ababneh, "On capacity improvement of optical free space chip-to-chip interconnects," Electrical Engineering, vol. 89, no. 6, pp. 451-456, May 2007, doi: 10.1007/s00202-006-0028-0.

[8] S. Tang, R. T. Chen, L. Garrett, D. Gerold, and M. M. Li, "Design limitations of highly parallel free-space optical interconnects based on arrays of vertical cavity surface-emitting laser diodes, microlenses, and photodetectors," Journal of Lightwave Technology, vol. 12, no. 11, pp. 1971-1975, 1994, doi: 10.1109/50.336062.

[9] A. G. Kirk, D. V. Plant, M. H. Ayliffe, M. Châteauneuf, and F. Lacroix, "Design rules for highly parallel free-space optical interconnects," IEEE Journal on Selected Topics in Quantum Electronics, vol. 9, no. 2, pp. 531-547, Mar. 2003, doi: 10.1109/JSTQE.2003.812482.

[10] M. Grabherr, "New applications boost VCSEL quantities: recent developments at Philips," in Vertical-Cavity Surface-Emitting Lasers XIX, Mar. 2015, vol. 9381, doi: 10.1117/12.2081790.

[11] A. Larsson, "Advances in VCSELs for communication and sensing," IEEE Journal on Selected Topics in Quantum Electronics, vol. 17, no. 6, pp. 1552-1567, Nov. 2011, doi: 10.1109/JSTQE.2011.2119469.

[12] J. Tatum, "VCSEL proliferation," in Vertical-Cavity Surface-Emitting Lasers XI, Feb. 2007, vol. 6484, doi: 10.1117/12.715082.

[13] J.-F. Seurin et al., "High-efficiency VCSEL arrays for illumination and sensing in consumer applications," in Vertical-Cavity Surface-Emitting Lasers XX, Mar. 2016, vol. 9766, doi: 10.1117/12.2213295.

[14] N. Mukoyama, H. Otoma, J. Sakurai, N. Ueki, and H. Nakayama, "VCSEL array-based light exposure system for laser printing," in Vertical-Cavity Surface-Emitting Lasers XII, Feb. 2008, vol. 6908, doi: 10.1117/12.768479.

[15] N. Al-Ababneh, "Crosstalk in misaligned free space optical interconnects: modelling and simulation," International Journal of Electrical and Computer Engineering (IJECE), vol. 9, no. 3, pp. 1620-1629, Jun. 2019, doi: 10.11591/ijece.v9i3.pp1620-1629.

[16] N. Al-Ababneh and A. Al Jumah, "Analytical models for channel crosstalk in short-range free space optical interconnects," Optical Engineering, vol. 51, no. 11, Nov. 2012, doi: 10.1117/1.OE.51.11.115401.

[17] K. Wang, A. Nirmalathas, C. Lim, E. Skafidas, and K. Alameh, "Experimental demonstration of $3 \times 310 \mathrm{~Gb} / \mathrm{s}$ reconfigurable free space optical card-to-card interconnects," Optics Letters, vol. 37, no. 13, pp. 2553-2555, Jun. 2012, doi: 10.1364/ol.37.002553.

[18] M. A. Neifeld and R. K. Kostuk, "Error correction for free-space optical interconnects: space-time resource optimization," Applied Optics, vol. 37, no. 2, pp. 296-307, Jan. 1998, doi: 10.1364/ao.37.000296.

[19] R. Wang, A. D. Rakić, and M. L. Majewski, "Analysis of lensless free-space optical interconnects based on multi-transverse mode vertical-cavity-surface-emitting lasers," Optics Communications, vol. 167, no. 1, pp. 261-271, Aug. 1999, doi: 10.1016/S0030-4018(99)00311-9.

[20] R. Wang, A. D. Rakić, and M. L. Majewski, "Design of microchannel free-space optical interconnects based on vertical-cavity surface-emitting laser arrays," Applied Optics, vol. 41, no. 17, pp. 3469-3478, Jun. 2002, doi: 10.1364/ao.41.003469.

[21] W. Jun, Z. Ge, and H. Weiwu, "An efficient error control scheme for chip-to-chip optical interconnects," in Proceedings - IEEE International Symposium on Circuits and Systems, May 2007, pp. 3712-3715, doi: 10.1109/iscas.2007.378649.

[22] N. Al-Ababneh and S. Tawalbeh, "Optimizing bandwidth density in free space optical interconnects under the use of error correcting codes," Optical and Quantum Electronics, vol. 46, no. 2, pp. 271-282, Aug. 2014, doi: 10.1007/s11082-013-9758-1.

[23] J. Faucher, M. B. Venditti, and D. V. Plant, "Application of parallel forward-error correction in two-dimensional optical-data links," Journal of Lightwave Technology, vol. 21, no. 2, pp. 466-475, Feb. 2003, doi: 10.1109/JLT.2003.808762.

[24] N. Bonneux and A. B. J. Kuijlaars, "Exceptional Laguerre Polynomials," Studies in Applied Mathematics, vol. 141, no. 4, pp. 547-595, Jan. 2018, doi: 10.1111/sapm.12204.

[25] M. Z. Konyar and S. Öztürk, "Reed Solomon coding-based medical image data hiding method against salt and pepper noise," Symmetry, vol. 12, no. 6, Jun. 2020, doi: 10.3390/sym12060899.

[26] H. Aldiabat and A. Alhubaishi, "Performance analysis of dual-branch selection combining technique over the generalized Alpha$\mathrm{Mu}$ fading channels," Indonesian Journal of Electrical Engineering and Computer Science (IJEECS), vol. 22, no. 2, pp. 1024-1031, May 2021, doi: 10.11591/ijeecs.v22.i2.pp1024-1031.

[27] D. J. C. Shu Lin, Error Control Coding: Fundamentals and Applications. Pearson-Prentice Hall, 2014. 


\section{BIOGRAPHIES OF AUTHORS}

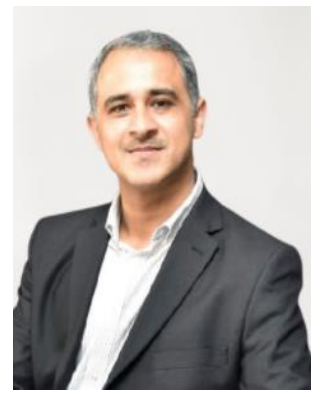

Hasan Aldiabat (iD S SC P is an assistant professor with the department of telecommunications engineering at Yarmouk University in Jordan. He received his B. Sc and M. Sc degrees in electrical engineering from Jordan University of Science and Technology in 2009 and 2011, respectively. He received his doctoral of engineering degree in electrical engineering from the University of Minnesota, Minneapolis, USA in 2019. His research interests are in free space optical interconnects, signal processing, and wireless communications. He can be contacted at email: hasan.aldiabat@yu.edu.jo.

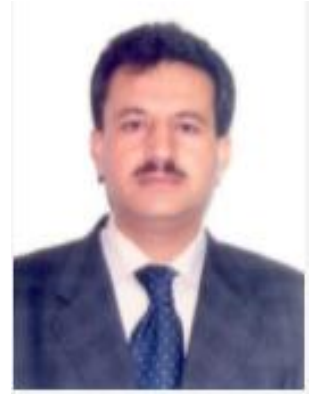

Nedal Al-Ababneh (iD) SC P received his BSc and MSc degrees in electrical engineering from Jordan University of Science and Technology in 1993 and 1996, respectively. He received his doctor of engineering degree in electrical engineering from the University of Massachusetts-Lowell, USA in 2004. He is currently a full professor in the Department of Electrical Engineering at Jordan University of Science and Technology. His research interests are in free-space optical interconnects. He can be contacted at email: nedalk@just.edu.jo. 